\title{
Negative effective permeability metamaterial using multi-layer ceramic capacitors
}

\author{
Thanh Binh Nguyen ${ }^{1}$, Naoyuki Kinai ${ }^{1}$, Naobumi Michishita ${ }^{1 a)}$, \\ Hisashi Morishita ${ }^{1}$, Teruki Miyazaki ${ }^{2}$, and Masato Tadokoro ${ }^{2}$ \\ ${ }^{1}$ Graduate School of Science and Engineering, National Defense Academy, \\ 1-10-20 Hashirimizu, Yokosuka, Kanagawa 239-8686, Japan \\ 2 The Yokohama Rubber Co., Ltd., \\ 2-1 Oiwake, Hiratsuka, Kanagawa 254-8601, Japan \\ a)naobumi@nda.ac.jp
}

\begin{abstract}
Multi-layer ceramic capacitors (MLCCs) have been employed for miniaturizing the unit cell structure of metamaterials. In addition, specific absorption rate suppression methods and thin wave absorbers based on metamaterials composed of MLCCs have been reported. This paper proposes a negative effective permeability metamaterial using MLCCs. Firstly, the effective permittivity and permeability of the unit cell are obtained from simulated $S$-parameters and compared with the dispersion characteristics. Next, transmission $S_{21}$ characteristic of the stacked metamaterial structure composed of the MLCCs is analyzed and then compared with the measurement result.
\end{abstract}

Keywords: multi-layer ceramic capacitor, negative effective permeability, metamaterial

Classification: Antennas and Propagation

\section{References}

[1] O. Luukkonen, F. Costa, C. R. Simovski, A. Monorchio, and S. A. Tretyakov, "A thin electromagnetic absorbers for wide incidence angles and both polarizations," IEEE Trans. Antennas Propag., vol. 57, no. 10, pp. 31193125, Oct. 2009. DOI:10.1109/TAP.2009.2028601

[2] S. A. Tretyakov and S. I. Maslovski, "Thin absorbing structure for all incidence angles based on the use of a high-impedance surface," Microw. Opt. Technol. Lett., vol. 38, pp. 175-178, Aug. 2003. DOI:10.1002/mop.11006

[3] D. Zhirihin, C. Simovski, P. Belov, and S. Glybovski, "Mushroom highimpedance metasurfaces for perfect absorption at two angles of incidence," IEEE Antennas Wireless Propag. Lett., vol. 16, pp. 2626-2629, Aug. 2017. DOI: 10.1109/LAWP.2017.2736506

[4] A. Toujo, T. Fujii, C. Kato, Y. Sakabe, and M. Kitano, "Possibility of synthesizing metamaterials with multilayer ceramic capacitors," IEICE General Conf., C-2-81, Mar. 2008 (in Japanese).

[5] A. Toujo, T. Fujii, K. Kageyama, H. Takagi, and M. Kitano, "New resonance mode of MLCC - Application to metamaterial," IEICE Technical Report, 
MW2008-149, pp. 19-24, Dec. 2008 (in Japanese).

[6] A. Toujo, "SAR reduction method by near field control," IEICE Trans. Electron., vol. J99-C, no. 9, pp. 417-424, Sept. 2016 (in Japanese).

[7] A. Toujo, "SAR reduction method using metamaterial," IEICE Society Conf., BCS-1-3, Sept. 2014 (in Japanese).

[8] N. Michishita, T. Miyazaki, M. Tadokoro, and H. Morishita, "Thin wave absorber using metasurface composed of multi-layer ceramic capacitors," IEICE Technical Report, AP2017-17, pp. 85-88, Apr. 2017 (in Japanese).

[9] Y. Konishi, "Practical microwave technology theory and practice," Nikkan Kogyo Shimbun, vol. 4, pp. 264-266, 2001 (in Japanese).

\section{Introduction}

Currently, antennas are being used in various devices such as mobile phones, and to reduce the influence of antennas on the human body and suppress unnecessary electromagnetic waves, metamaterials with periodic structures of metal patches [1], mushroom structures $[2,3]$ have been proposed. However, if the size of a unit cell of metamaterials is large, it will be impossible to arrange the cells efficiently when loading them onto such a narrow object or concave-convex surface. Therefore, the size of a unit cell is required to miniaturize.

In previous studies, the possibility of synthesizing metamaterials with multilayer ceramic capacitors (MLCCs) $[4,5]$ has been reported. It has been confirmed that there is a band showing negative permeability by using MLCCs. In the frequency band which shows the negative permeability, because the magnetic flux penetrates between the internal electrodes of the MLCC, the current circulates in a loop shape and becomes an equivalent circuit similar to the split ring resonator (SRR) [6]. Therefore, the SAR (Specific Absorption Rate) suppression method $[6,7]$ and thin wave absorbers [8] using MLCCs have been studied, leading to the expectation that it can be used for miniaturizing the unit cell and loading onto the narrow object. Also, MLCC has the added merits of being easy to obtain and inexpensive.

In this paper, we design a negative permeability metasurface with two MLCCs located on the dielectric substrate. By using simulated $S$-parameters, the effective permittivity and permeability of the unit cell are extracted and compared with the dispersion characteristics. Next, the $S_{21}$ characteristic of the stacked metamaterial structure made up of the MLCCs is analyzed and compared with the measurement result.

\section{The proposed unit cell and stacked metamaterial structures}

The unit cell structure proposed in this paper is shown in Fig. 1(a). Two MLCCs are located between two strip conductors with $0.5 \mathrm{~mm}$ width on a dielectric substrate whose thickness is $0.75 \mathrm{~mm}$ with $\varepsilon_{b}=3.7$. The cutoff frequency of two strip conductors is less than $10 \mathrm{GHz}$ and it is lower than the design frequency of the negative permeability metasurface proposed in this paper. So, the negative permeability characteristic is not affected. Fig. 1(b) shows MLCC structure. The external dimensions of the MLCC are $1.25 \mathrm{~mm} \times 0.85 \mathrm{~mm} \times 2.2 \mathrm{~mm}$, and relative permit- 


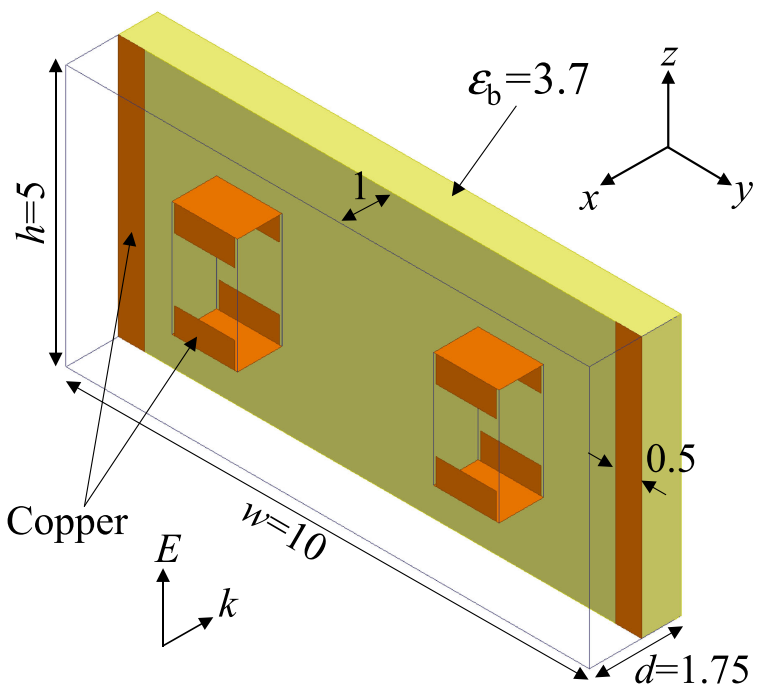

(a)
[Unit: $\mathrm{mm}]$

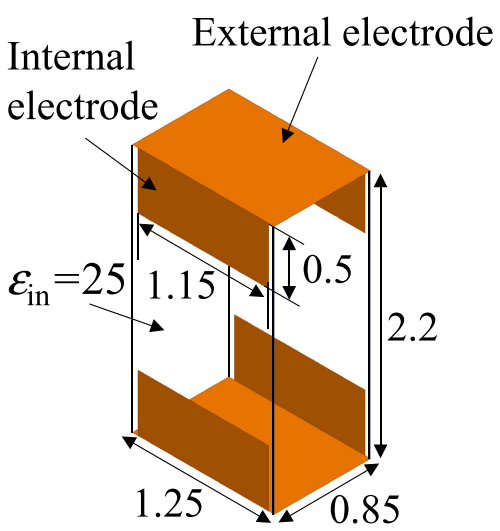

(b)

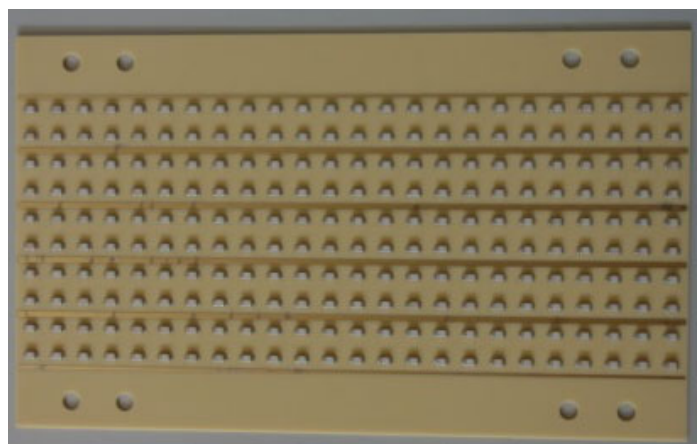

(c)

Fig. 1. (a) Configuration of the proposed unit cell structure. (b) Configuration of MLCC structure. (c) Prototype structure of stacked metamaterial

tivity of the internal dielectric material is $\varepsilon_{i n}=25$. Four internal copper electrodes of length $0.5 \mathrm{~mm}$ and width $1.15 \mathrm{~mm}$ are modeled. Ansoft HFSS ver.13 with the finite-element method has been employed for simulation.

Fig. 1(c) shows the prototype structure of stacked metamaterial made up of the MLCCs. The prototype structure was fabricated by stacking five layers of metasurface with the unit cell structure as shown in Fig. 1(a). It was arranged in an array of five cells in the $y$ axis direction and twenty-four cells in the $z$ axis direction. And the gap between the respective dielectric substrates is $1 \mathrm{~mm}$.

\section{Effective permittivity and permeability of proposed unit cell structure}

Fig. 2(a) shows the dispersion characteristics of the unit cell by eigenmode analysis. The bandgap occurs at the frequency band between $22.3 \mathrm{GHz}$ to $22.8 \mathrm{GHz}$.

In addition, the effective permittivity and permeability of the proposed unit cell are extracted from $S$-parameters simulation results [9]. Fig. 2(b), 2(c) show the real and imaginary parts of the effective permittivity and permeability from 20 to $26 \mathrm{GHz}$. The real part of the effective permittivity is always positive within the given frequency band. The imaginary part of effective permittivity and permeability 


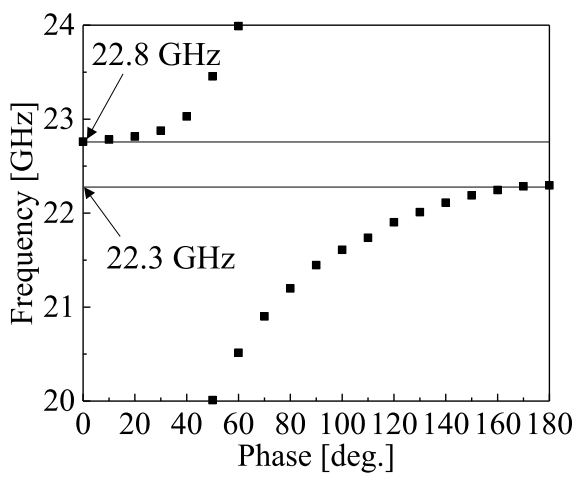

(a)

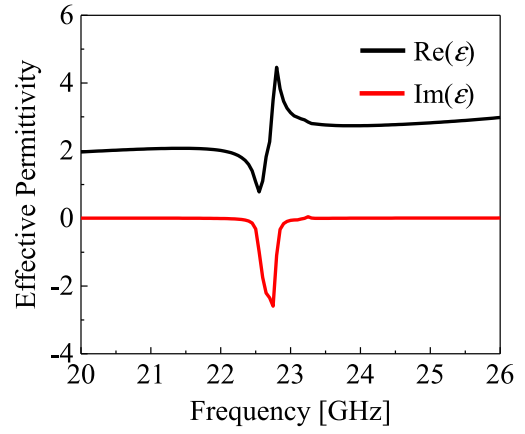

(b)

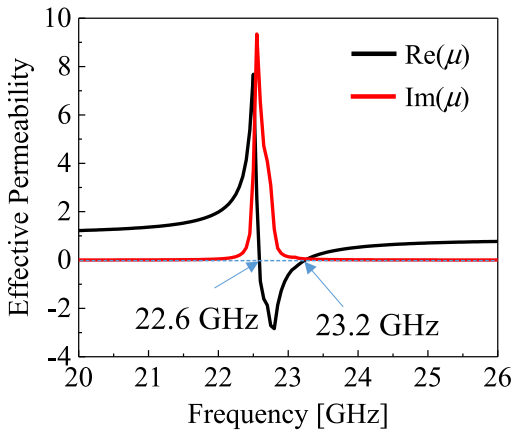

(c)

Fig. 2. (a) Dispersion characteristics. (b) Effective permittivity and (c) effective permeability of the proposed unit cell

is near to zero except at resonant frequency. The real part of the effective permeability shows an abrupt change in the value around the resonant frequency and becomes negative from $22.6 \mathrm{GHz}$ to $23.2 \mathrm{GHz}$. The frequency band shifts higher in comparison with that for the dispersion characteristics.

\section{Comparison between the simulation result and measurement result}

The proposed unit cell structure as shown in Fig. 1(a) is stacked in array of five layers in $x$ axis direction and transmission $S_{21}$ characteristics are analyzed. The measurement result is also shown in Fig. 3. It was found that the stopband of the measurement result is confirmed at around $23 \mathrm{GHz}$ but it is larger than the stopband of simulation result.

And to address the differences between the simulation result and measurement result, the influence of the variation of the MLCC is investigated. From the measurement result, the resonant frequencies of the MLCCs are varied at 21.6 GHz, $22.0 \mathrm{GHz}, 22.3 \mathrm{GHz}$ and $23.1 \mathrm{GHz}$. To realize these resonant frequencies, the length of internal electrodes of the MLCCs are adjusted to $0.540 \mathrm{~mm}$, $0.528 \mathrm{~mm}, 0.515 \mathrm{~mm}$ and $0.490 \mathrm{~mm}$, respectively. The $S_{21}$ characteristics of the five layers stacked structure with these four length and the initial length of $0.500 \mathrm{~mm}$ are also analyzed. As shown in Fig. 3, the stopband of simulation result becomes wider and almost agrees with the measurement result by considering the variation of the MLCC. 


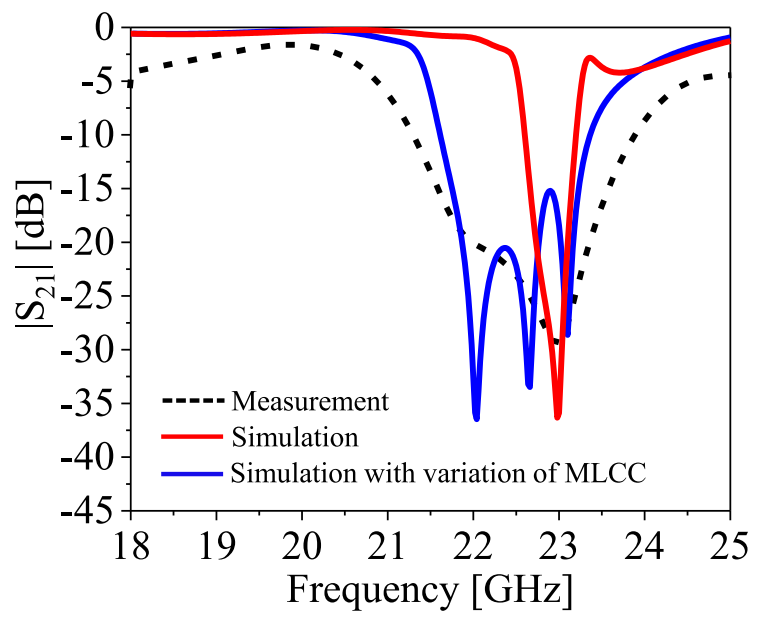

Fig. 3. Comparison between the simulation result and measurement result

\section{Conclusion}

In this paper, the negative permeability material using MLCCs has been proposed. The effective permittivity and permeability of the proposed unit cell structure were extracted from simulated $S$-parameters. The results of the comparison with the dispersion characteristics showed that the frequency band where the effective permeability becomes negative shifted higher with the bandgap. Also, the prototype structure was fabricated and tested experimentally. Measurement result showed that the stopband is confirmed at around $23 \mathrm{GHz}$ but it was larger than the stopband of the simulation result. And to address this, the influence of the variation of MLCC was investigated. It was found that by considering the variation of the MLCC, the simulation result almost agreed with the measurement result.

\section{Acknowledgements}

A part of this work was supported by JSPS KAKENHI Grant Number 25820168. 